\title{
The importance of the implant quality in APBI - Gliwice experience. Dosimetric evaluation
}

\author{
Agnieszka Cholewka, MSc', Marta Szlag, MSc, PhD', Brygida Białas, MD, PhD², Sylwia Kellas-Śleczka, MD, PhD², \\ Krzysztof Ślosarek, MSc, PhD, Prof! \\ 'Department of Radiotherapy and Brachytherapy Planning, ${ }^{2}$ Brachytherapy Department, Maria Sklodowska-Curie Memorial Cancer Center \\ and Institute of Oncology, Gliwice, Poland
}

\begin{abstract}
Purpose: This study covers four years of our clinical trials to improve implant quality in multicatheter Accelerated Partial Breast Irradiation (APBI). The progress made in dosimetric and volumetric parameters of the treatment plans was evaluated.

Material and methods: One hundred and ninety-one women, for whom treatment plans were made based on three dimensional imaging, were selected for the study. To evaluate progress made in our APBI procedure, following parameters and indices were taken into account: percentage of the target volume receiving the reference dose $\left(\mathrm{PTV}_{\text {ref }}\right)$, minimum dose in the target volume expressed as a percentage of reference dose $\left(\mathrm{PTV}_{\min }\right)$, dose homogeneity index (DHI) and conformity index (COIN). Additionally the plan quality index was calculated for every group as the sum of mean values of four evaluated parameters.

Results: PTV ${ }_{\text {ref }}$ have increased from the mean value of $83.4 \%$ at the beginning to $94.8 \%$ recently. The maximum value equals $95.4 \%$. The same trend can be observed with $\mathrm{PTV}_{\min }$ value which has been improved from $51.7 \%$ to $70.1 \%$ maximally. DHI and COIN mean values present the similar progress. DHI value increased from 0.53 level to 0.68 and COIN from 0.58 in 2009 to 0.74 . Plan quality index has increased from 2.46 in 2009 to 3.06 recently.

Conclusions: The implant quality is crucial for the accurate dose distribution. This paper shows the progress we've made in APBI procedure to improve implant quality. Nowadays our implant technique based on three-dimensional $\mathrm{CT}$ imaging results in acceptable dose distributions.

Key words: accelerated partial breast irradiation, dosimetric analysis, implant quality, interstitial brachytherapy.

\section{Purpose}

The role of accelerated partial breast irradiation (APBI) for patients with early stage breast cancer has gained popularity as an alternative to the conventional whole breast irradiation (WBI) with external beam radiation [1-12]. In recent years, new single-entry breast brachytherapy devices have emerged offering simpler usage both for a patient and a physician $[1,13]$. However, multicatheter interstitial brachytherapy with flexible catheters located around the lumpectomy cavity, as the oldest one, has the longest experience. There are many studies presenting multicatheter interstitial APBI results in literature [1-14].

Because of the growing interest in APBI methods, guidelines and recommendations have been recently formulated [3]. In 2009, the American Society for Radiation Oncology (ASTRO) has published their statement on the use of APBI [15]. In the same year, the Groupe Européen de Curiethérapie European Society for Therapeutic Radiology and Oncology (GEC-ESTRO) Breast Cancer Working Group has published the European guidelines for patient selection for APBI with interstitial brachytherapy (BT) [16].
In the past, two-dimensional radiography based imaging techniques were used in order to evaluate the dose distribution. However, in multicatheter APBI, the precise target definition and implant quality are crucial for accurate and conformal delivery of the prescribed dose to the planning target volume (PTV) with simultaneous sparing of normal tissue and organs at risk $[2,7,8,11,17,18]$. Three-dimensional imaging is therefore essential [3,18-22].

Since 2006, APBI as an adjuvant technique after breast conserving surgery has been used in Brachytherapy Department in Gliwice. From the beginning till the end of 2008, fluoroscopic films (two-dimensional, 2D imaging) were taken for both treatment planning and pre-planning procedures. Pre-planning was used to evaluate the implant geometry with respect to the target. The two-dimensional X-ray imaging system IBU-D (Integrated Brachytherapy Unit by Nucletron, an Elekta company, Elekta AB, Stockholm, Sweden) was used. Two orthogonal films were taken before insertion of needles to evaluate the location of the surgical clips, and after the implantation procedure to assess the geometric quality of the implant. Treatment planning, based on orthogonal films was per-

Address for correspondence: Agnieszka Cholewka, MSc, Department of Radiotherapy and Brachytherapy Received: 05.11 .2013 Planning, Maria Sklodowska-Curie Memorial Cancer Center and Institute of Oncology, 15 Wybrzeże Armii Accepted: 04.12 .2013 Krajowej Street, 44-101 Gliwice, Poland, phone: +48 32278 92 53, e-mail: agnieszka.cholewka@gmail.com Published: 31.12 .2013 
formed with PLATO BPS v.14.3.3 planning system (Nucletron).

In 2009, three-dimensional computed tomography (CT) based treatment planning was introduced to APBI method. Treatment plans based on CT scans were created in the Oncentra MasterPlan v.3.1 planning system (Nucletron). The possibility of target delineating and estimating the target coverage with prescribed dose, as well as evaluating the maximum dose in organs at risk appeared.

This study covers four years of our clinical trials with multicatheter APBI based on three dimensional imaging. Dosimetric and volumetric parameters of the treatment plans were evaluated.

\section{Material and methods}

Between June 2006 and December 2012, 232 patients were treated with multicatheter APBI at the Brachytherapy Department in Memorial Cancer Center - Institute in Gliwice. One hundred and ninety-one of them, for whom treatment plans were made based on three dimensional imaging, were selected for the study. Since January 2009 till December 2012, eight separated groups have been created - one group every six months. We have used this approach to study the systematic improvement of the APBI technique with respect to the dosimetric parameters of the treatment plans.

All patients underwent breast conserving surgery. The cavity wall, after lumpectomy, was marked with the surgical titanium clips. The flexible catheters were implanted into the breast through a template, in a triangular pattern. Inter-catheter distance, number of planes, and catheters were adjusted with respect to the size and location of the target defined as a marked lesion with $0.5-2 \mathrm{~cm}$ margins. In the analyzed treatment plans, two or threeplane implants were used for all patients with $1.0-1.6 \mathrm{~cm}$ separation between the catheters.

Three-dimensional pre-planning method was performed with the Simulix Evolution Simulator (Nucletron). We have been trying to focus on pre-planning procedure emphatically all the time, as we consider it underlies as

Table 1. Implant characteristics, volume, and dose parameters

\begin{tabular}{lcc} 
Parameter & Mean value & Range \\
\hline Number of catheters & 14 & $7-18$ \\
\hline Number of planes & 3 & $2-5$ \\
\hline $\mathrm{V}_{\text {PTV }}\left[\mathrm{cm}^{3}\right]$ & 71.6 & $17.4-226.5$ \\
\hline $\mathrm{PTV}_{\text {ref }}[\%]$ & 93.7 & $69.9-99.1$ \\
\hline $\mathrm{D}_{90}[\%]$ & 106.0 & $66-122.9$ \\
\hline $\mathrm{PTV}$ & 66.4 & $23.5-87.8$ \\
\hline $\mathrm{COIN}$ & 0.69 & $0.37-0.84$ \\
\hline DHI & 0.66 & $0.40-0.78$ \\
\hline Skin $\mathrm{D}_{\max }[\%]$ & 43.4 & $17.0-70.0$ \\
\hline Ipsilateral lung $\mathrm{D}_{\max }[\%]$ & 51.1 & $4.6-87.5$ \\
\hline Ipsilateral lung $\mathrm{D}_{2}[\%]$ & 41.2 & $13.0-68.3$ \\
\hline Ipsilateral lung $\mathrm{D}_{10}[\%]$ & 31.7 & $10.0-55.0$
\end{tabular}

the acceptable treatment plan. At the beginning of our trials with three-dimensional pre-planning, we performed cone beam CT acquisition to evaluate the surgical clips location. Then we decided to perform the second acquisition after inserting one plane of the needles to evaluate the position of the needles in relation to the marked lesion. Additional needles were inserted if necessary. In some cases, we decided to perform one more acquisition to make sure the needles positions were correct. Finally, the flexible plastic catheters were implanted into the breast to replace metal needles. Immediately after the implantation, computed tomography with Somatom Sensation Open 20 (Siemens AG, Munich, Germany) was performed for treatment planning purpose (catheter reconstruction, definition of planning target volume, and organs at risk volumes and plan evaluation). Treatment scheme was the same for every patient. The delivered dose was $32 \mathrm{~Gy}$ in eight fractions (4 Gy per fraction).

Treatment plans in each group were evaluated based on the dosimetric and volumetric parameters derived from the cumulative dose volume histograms (cDVH). Percentage of the target volume receiving the reference dose $\left(\mathrm{PTV}_{\text {ref }}\right)$, minimum dose in the target volume expressed as a percentage of reference dose $\left(\mathrm{PTV}_{\min }\right)$, dose homogeneity index (DHI), and conformity index (COIN) has been selected for analysis. According to the definition of DHI $=1-\left(V_{150} / V_{\text {ref }}\right), V_{\text {ref }}$ and $V_{150}$ quantify volumes receiving the reference, and 1.5 times of the reference dose, respectively. The COIN determines coverage of the target by the reference isodose, including unwanted irradiation of normal tissues and is defined as $\mathrm{COIN}=\left(\mathrm{PTV}_{100} / \mathrm{V}_{\text {ref }}\right)\left(\mathrm{PTV}_{100} / \mathrm{V}_{\mathrm{PTV}}\right) . \mathrm{PTV}_{100}$ is the volume of the target receiving the prescribed dose. Mean values with standard error of the parameters were computed for every group.

Statistical analysis was done with Statistica v.10 (StatSoft, Inc., Tulsa, USA). Differences with a $p$ value $<0.05$ were regarded as significant. The Kruskall-Wallis nonparametric test was used to compare values in groups.

In order to present the total progress made in APBI procedure, we proposed a plan quality index (PQI) defined as a sum of mean values of four evaluated param-

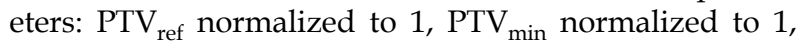
DHI, and COIN indexes. PQI was calculated for every group of patient.

\section{Results}

Implant characteristics, dose-volume parameters and critical organs doses are shown in Table 1. The mean volume of the PTV was $71.6 \mathrm{~cm}^{3}$. Generally, three-plane implants were made with the mean value of catheters equal 14. The mean values of target coverage with prescribed dose $\left(\mathrm{PTV}_{\text {ref }}\right)$ was $93.7 \%$, minimum dose in the target volume $\left(\mathrm{PTV}_{\min }\right)$ was $66.4 \%$, and the dose encompassing $90 \%$ of the PTV $\left(D_{90}\right)$ was $106 \%$. The mean values of implant characterizing indices were acceptable, and they came to 0.69 for conformity index (COIN) and 0.66 for homogeneity index (DHI).

The mean value of maximum skin dose was $43.3 \%$ of the prescribed dose. The mean value of ipsilateral lung 
Table 2. Mean values of parameters with standard deviations for groups presenting the consecutive periods of time

\begin{tabular}{lcccccc} 
& Number of cases & PTV $_{\text {ref }}$ [\%] & PTV $_{\min }[\%]$ & DHI & COIN & PQI \\
\hline [I] 01-06.2009 & 8 & $83.4 \pm 9.4$ & $51.7 \pm 13.7$ & $0.53 \pm 0.09$ & $0.58 \pm 0.13$ & $2.46 \pm 0.45$ \\
\hline [II] 07-12.2009 & 15 & $91.1 \pm 5.7$ & $64.0 \pm 13.0$ & $0.59 \pm 0.07$ & $0.57 \pm 0.08$ & $2.71 \pm 0.34$ \\
\hline [III] 01-06.2010 & 21 & $93.3 \pm 5.3$ & $68.3 \pm 14.9$ & $0.67 \pm 0.08$ & $0.62 \pm 0.10$ & $2.90 \pm 0.38$ \\
\hline [IV] 07-12.2010 & 22 & $95.4 \pm 1.7$ & $70.1 \pm 8.8$ & $0.68 \pm 0.05$ & $0.60 \pm 0.06$ & $2.93 \pm 0.22$ \\
\hline [V] 01-06.2011 & 24 & $94.8 \pm 3.7$ & $68.9 \pm 12.5$ & $0.66 \pm 0.08$ & $0.71 \pm 0.04$ & $3.01 \pm 0.28$ \\
\hline [VI] 07-12.2011 & 37 & $93.9 \pm 3.1$ & $65.4 \pm 8.6$ & $0.67 \pm 0.06$ & $0.75 \pm 0.04$ & $3.01 \pm 0.22$ \\
\hline [VII] 01-06.2012 & 34 & $94.7 \pm 2.9$ & $67.3 \pm 9.6$ & $0.68 \pm 0.06$ & $0.76 \pm 0.06$ & $3.06 \pm 0.25$ \\
\hline [VIII] 07-12.2012 & 30 & $94.8 \pm 1.7$ & $66.0 \pm 10.7$ & $0.68 \pm 0.07$ & $0.74 \pm 0.05$ & $3.02 \pm 0.24$ \\
\hline p (Kruskal-Wallis) & - & 0.0015 & 0.0115 & 0.0000 & 0.0000 & -
\end{tabular}

dose to 2 cc volume was $41.2 \%$ of the prescribed dose, and the lung dose to a $10 \mathrm{cc}$ volume was $31.7 \%$; however, the maximum dose for ipsilateral lung was $51.1 \%$ on average. Recently, the maximum dose for the ribs surface is evaluated, though it is not taken into consideration in this study.

Table 2 and the Figures 1-4 show the evaluated parameters divided into groups presenting the consecutive periods of time. The data present mean value of analyzed parameters with standard deviations. The differences between groups are statistically significant, as $p$ value is lower than 0.05 for every parameter evaluated.

$\mathrm{PTV}_{\text {ref }}$ have increased from mean value of $83.4 \%$ at the beginning to recent $94.8 \%$. The maximum value equals $95.4 \%$ (Fig. 1). The same trend can be observed with $\mathrm{PTV}_{\min }$ value, which has been improved from $51.7 \%$ to maximally $70.1 \%$ (Fig. 2). DHI and COIN mean values (Figs. 3 and 4) present the similar progress. DHI value increased from 0.53 level to 0.68 , and COIN from 0.58 in 2009 to 0.74 lately.

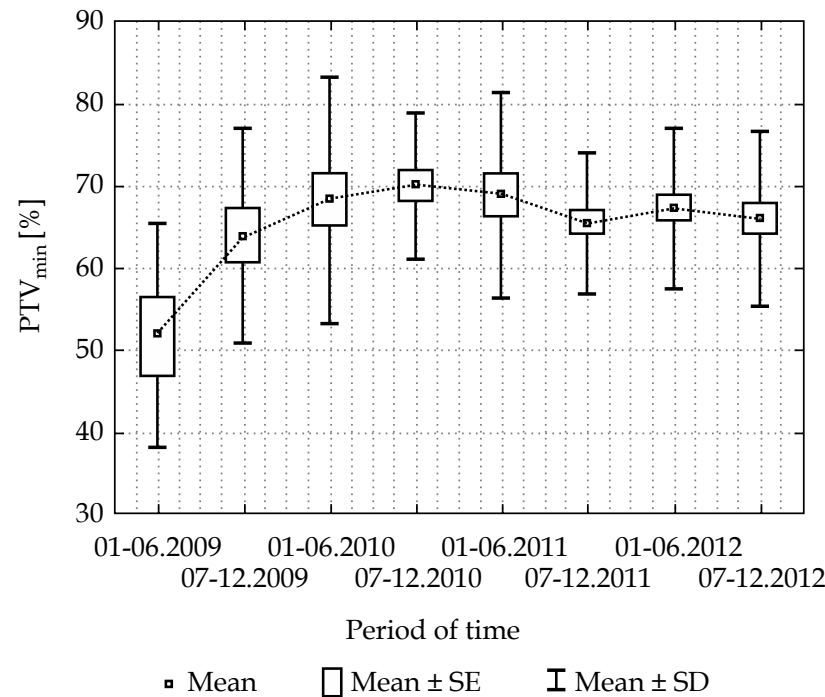

Fig. 2. Mean value of $P T V_{\min }$ in the consecutive periods of time

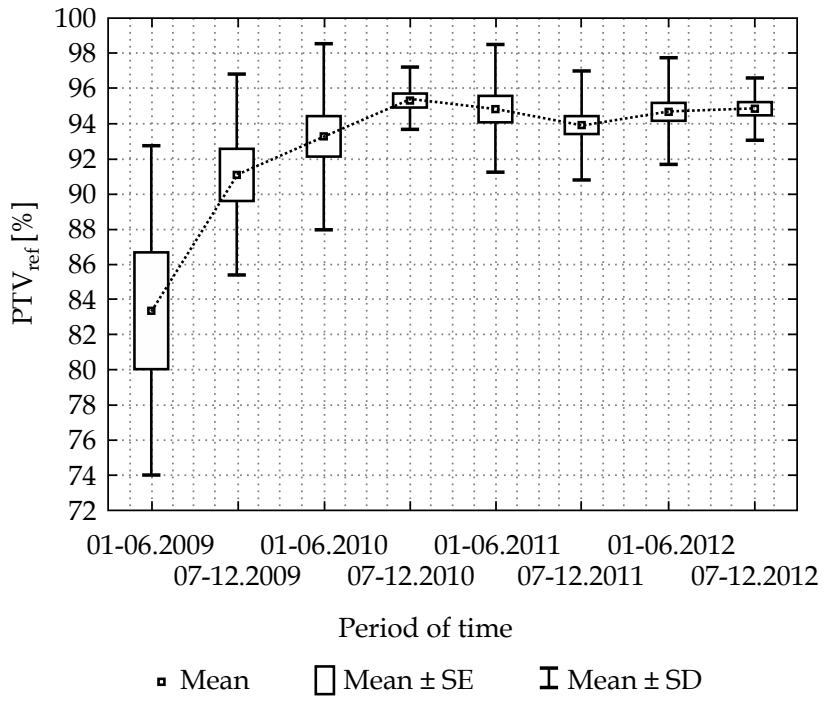

Fig. 1. Mean value of $\mathrm{PTV}_{\text {ref }}$ in the consecutive periods of time

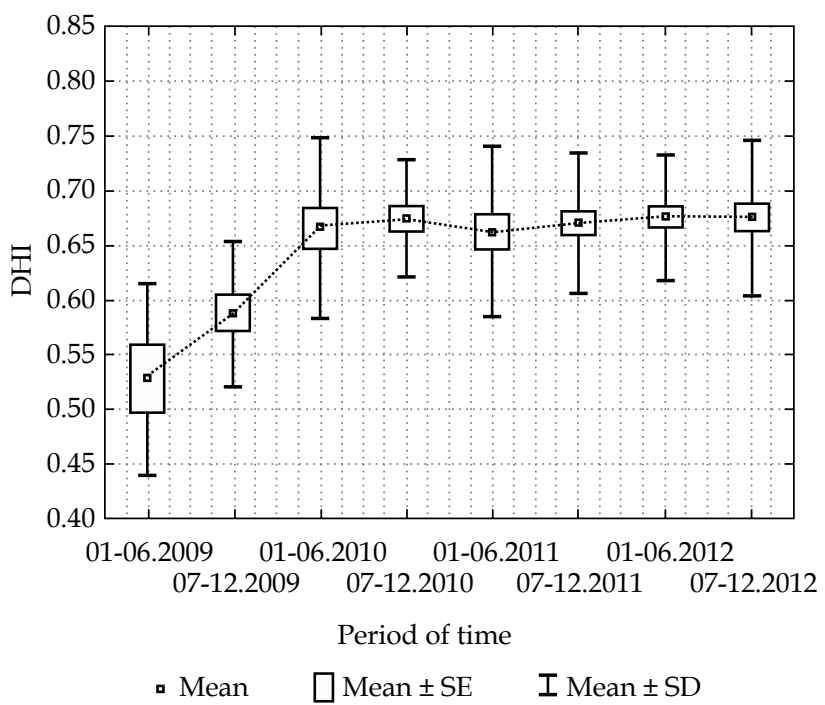

Fig. 3. Mean value of DHI in the consecutive periods of time 


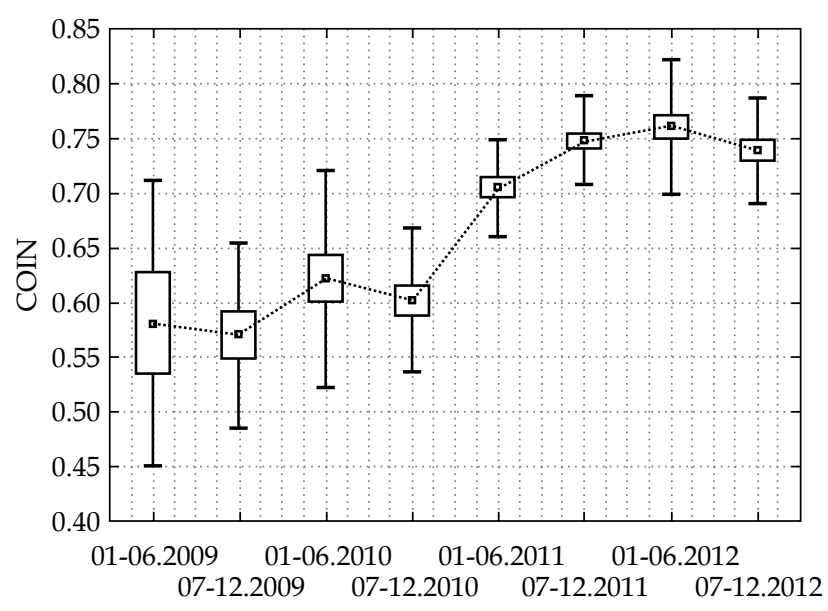

Period of time

- Mean $\square$ Mean+SE I Mean+SD

Fig. 4. Mean value of COIN in the consecutive periods of time

\section{Discussion}

In multicatheter interstitial APBI, the precise target definition and implant quality are crucial for accurate and conformal delivery of the prescribed dose to the PTV, with simultaneous sparing of normal tissue and organs at risk $[2,7,8,11,17,18]$. Our results confirm these findings. From the very beginning, APBI technique in all over the world is being constantly improved in order to achieve good results with acceptable dosimetric parameters.

To improve dose distributions, dose optimization algorithms can be used. However, modification of the dwell times may produce overdose areas inside the treated volume [3,22,24]. Kestin et al. [20] have shown in their study that adding a few dwell positions improved target coverage from $87 \%$ to $97 \%$ of prescribed dose. Nevertheless, volume of tissue receiving $150 \%$ of the prescribed dose also increased.

From all factors that have influence the dose distribution, implant quality should be listed at the first place. We confirmed that improvement in implant quality increases the dose distribution conformity and homogeneity without extensive use of the optimization algorithms. That explains the requirement of use the accurate imaging method for treatment planning and pre-planning procedures. Two-dimensional orthogonal-based imaging has been replaced with CT-based three-dimensional [2,11,24]. CT-guided implantation improves dose distribution allowing the needles to be inserted precisely with respect to the target volume. Vicini et al. [19] demonstrated that CT-based dosimetry is needed to evaluate target coverage. Polgár and Major [26] emphasized that in modern brachytherapy, the treatment planning and plan evaluation have to be based on the 3D volume of the PTV, even though the use of a two-film localization technique allows the reconstruction of the catheters in three dimensions.

Many studies demonstrate beneficial effect of 3D imaging on dosimetric parameters of treatment plan, and discourage form using traditional two-dimensional implantation techniques for interstitial brachytherapy [19-21,

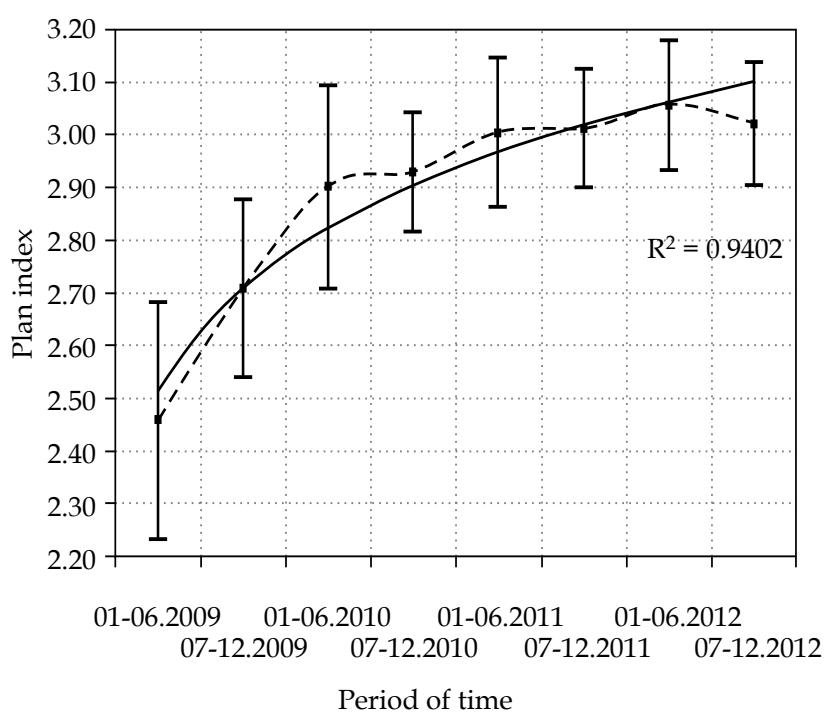

Fig. 5. Plan quality index value in the consecutive periods of time

$24,25]$. The accuracy of dose delivery can be significantly improved with the use of 3D imaging before or during implantation. Vicini et al. [19] has conduct a study in which they selected patients who underwent CT scanning after implant placement to evaluate executed dose distribution with respect to delineated target volume. A median of only $68 \%$ of this volume received $100 \%$ of the prescribed dose. Major et al. $[22,24,26]$ have compared dosimetric parameters achieved by replacing fluoroscopy-based needles implantation with CT-based one. All evaluated parameters have increased $\left(70 \%\right.$ vs. $91 \%$ for $\mathrm{PTV}_{100}, 0.35$ vs. 0.33 for DNR, 0.40 vs. 0.68 for COIN) and improved the quality of the implants significantly. Cuttino et al. [25] reported that changing traditional two-dimensional techniques to a CT-guided technique caused mean target coverage (defined as the percentage of PTV with $2 \mathrm{~cm}$ margin receiving $90 \%$ of the prescribed dose) to increase from $42 \%$ to $93 \%$, and mean DHI to increase from 0.77 to 0.82 .

Since three-dimensional preplanning has been introduced in our institute with the additional acquisition after inserting one plane of the needles, target coverage has improved significantly [2]. With such procedures we are able to gain treatment plan dosimetric parameters comparable to other institutions achievements. Recently, it has been about $95 \%$ for PTV $_{\text {ref }}, 66 \%$ for PTV $_{\text {min }}, 0.68$ for DHI, and 0.75 for COIN index.

In brachytherapy planning it is highly recommended to deliver at least the prescribed dose to the target with acceptable homogeneity and conformity of the dose distribution $[2,23]$. However, in some situations acceptable target coverage excludes satisfactory homogeneity and conformity of the dose distribution, so finding a balance between these parameters is required. Our results confirm these findings. It can be seen, for every parameter evaluated, that the mean value has reached the required level at one point, and then it has been oscillating around it. It means that the chosen parameter's value is higher in one period and lower in another one. However, higher value of one parameter usually effects lower value of another one during the treatment 
planning. That prompted us to calculate the plan quality index as the sum of mean values of four evaluated param-

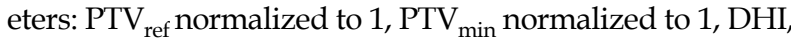
and COIN indexes. Figure 5 shows the plan quality index mean value in the consecutive periods of time.

From the beginning of three-dimensional era in our institute (two-dimensional era is not taken into account in this paper), the plan quality index has become higher. Plan quality index maximum value can be 4 . However, it is not possible to achieve it in practice. In our institute plan quality index value has increased from 2.46 to 3.06 , maximally. High correlation with the logarithmic trend curve with $\mathrm{R}^{2}$ comes to 0.94 can be observed. That proves how hard we try to improve dose distributions.

\section{Conclusions}

According to ESTRO and ASTRO recommendations, reporting dosimetric parameters of implant as well as surrounding organs exposure to risk are obligatory. However, there are still no guidelines determining the required level of some parameters in brachytherapy of breast cancer. It is highly recommended to deliver at least the prescribed dose to the target with acceptable homogeneity and conformity of the dose distribution. The implant quality is crucial for the accurate dose distribution. This paper shows the progress we've made in APBI procedure to improve implant quality. It can be seen that nowadays our implant technique based on three-dimensional CT imaging results in acceptable dose distributions.

\section{References}

1. Bloom ES, Kirsner S, Mason BE et al. Accelerated partial breast irradiation using the strut-adjusted volume implant single-entry hybrid catheter in brachytherapy for breast cancer in the setting of breast augmentation. Brachytherapy 2011; 10: 178-183.

2. Cholewka A, Szlag M, Ślosarek K et al. Comparison of 2Dand 3D-guided implantation in accelerated partial breast irradiation (APBI). J Contemp Brachytherapy 2009; 1: 207-210.

3. Major T, Polgár C, Lövey K et al. Dosimetric characteristics of accelerated partial breast irradiation with CT image-based multicatheter interstitial brachytherapy: A single institution's experience. Brachytherapy 2011; 10: 421-426.

4. Elliott RL, DeLand M, Head JF et al. Accelerated partial breast irradiation: Initial experience with the Intrabeam System. Surg Oncol 2011; 20: 73-79.

5. Cuttino LW, Todor D, Rosu M et al. A comparison of skin and chest wall dose delivered with multicatheter, Contura multilumen balloon, and Mammosite breast brachytherapy. Int J Radiat Oncol Biol Phys 2011; 79: 34-38.

6. Arthur D, Vicini F. Accelerated partial breast irradiation as a part of breast conservation therapy. J Clin Oncol 2005; 23: 1726-1735.

7. Kuerer HM, Julian TB, Strom EA et al. Accelerated Partial Breast Irradiation After Conservative Surgery for Breast Cancer. Ann Surg 2004; 239: 338-351.

8. Scanderbeg DJ, Yashar C, Rice R et al. Clinical implementation of a new HDR brachytherapy device for partial breast irradiation. Radioth Oncol 2009; 90: 36-42.

9. Major T, Niehoff P, Kovacs G et al. Dosimetric comparisons between high dose rate interstitial and MammoSite balloon brachytherapy for breast cancer. Radiother Oncol 2006; 79: 321-328.
10. Polgár C, Major T, Fodor J et al. High-dose-rate brachytherapy alone versus whole breast radiotherapy with or without tumor bed boost after breast-conserving surgery: seven-year results of a comparative study. Int J Radiat Oncol Biol Phys 2004; 60: 1173-1181.

11. Patel RR, Das RK. Image-guided breast brachytherapy: an alternative to whole-breast radiotherapy. Lancet Oncol 2006; 7: 407-415.

12. Belkacemi Y, Chauvet MP, Giard S et al. Partial breast irradiation as sole therapy for low risk breast carcinoma: Early toxicity, cosmesis and quality of life results of a MammoSite brachytherapy phase II study. Radioth Oncol 2009; 90: 23-29.

13. Sarin R. Partial-breast treatment for early breast cancer: Emergence of a new paradigm. Nat Clin Pract Oncol 2005; 2: 40-47.

14. Kushe R. Accelerated partial breast irradiation: moving forward. Amer Soc Breast Dis 2006; 4: 1-6.

15. Smith BD, Arthur DW, Buchholz TA et al. Accelerated partial breast irradiation consensus statement from the American Society for Radiation Oncology (ASTRO). Int J Radiat Oncol Biol Phys 2009; 74: 987-1001.

16. Polgár C, van Limbergen E, Pötter R et al. On behalf of the GEC-ESTRO Breast Cancer Working Group. Patient selection for accelerated partial-breast irradiation (APBI) after breast-conserving surgery: Recommendations of the Groupe Européen de Curiethérapie - European Society for Therapeutic Radiology and Oncology (GEC-ESTRO) Breast Cancer Working Group based on clinical evidence (2009). Radiother Oncol 2010; 94: 264-273.

17. Ott OJ, Hildebrandt G, Pötter R et al. Accelerated partial breast irradiation with multi-catheter brachytherapy: Local control, side effects and cosmetic outcome for 274 patients. Results of the German-Austrian multi-centre trial. Radioth Oncol 2007; 82: 281-286.

18. Vicini FA, Jaffray DA, Horwitz EM et al. Implementation of $3 \mathrm{D}$ virtual brachytherapy in the management of breast cancer: A description of a new method of interstitial brachytherapy. Int J Radiat Oncol Biol Phys 1998; 40: 629-635.

19. Vicini FA, Kestin LL, Edmundson GK et al. Dose-volume analysis for quality assurance of interstitial brachytherapy for breast cancer. Int J Radiat Oncol Biol Phys 1999; 45: 803-810.

20. Kestin LL, Jaffray DA, Edmundson GK et al. Improving the dosimetric coverage of interstitial high-dose-rate breast implants. Int I Radiat Oncol Biol Phys 2000; 46: 35-43.

21. Weed DW, Edmundson K, Vicini FA et al. Accelerated partial breast irradiation: A dosimetric comparison of three different techniques. Brachytherapy 2005; 4: 121-129.

22. Major T, Fodor J, Takacsi-Nagy Z et al. Evaluation of HDR interstitial breast implants planned by conventional and optimized CT-based dosimetry systems with respect to dose homogeneity and conformality. Strahlenther Onkol 2005; 181: 89-96.

23. Bovi J, Qi XS, White J et al. Comparison of three accelerated partial breast irradiation techniques: Treatment effectiveness based upon biological models. Radiother Oncol 2007; 84: 226-232.

24. Major T, Fröhlich G, Lövey K et al. Dosimetric experience with accelerated partial breast irradiation using image-guided interstitial brachytherapy. Radioth Oncol 2009; 90: 48-55.

25. Cuttino LW, Todor D, Arthur DW. CT-guided multi-catheter insertion technique for partial breast brachytherapy: reliable target coverage and dose homogeneity. Brachytherapy 2005; 4: 10-17.

26. Polgár C, Major T. Current status and perspectives of brachytherapy for breast cancer. Int J Clin Oncol 2009; 14: 7-24. 